\title{
RAIRO-RO
}

\section{Livres reçus à la bibliothèque de l'A.F.C.E.T.}

Revue française d'automatique, d'informatique et de recherche opérationnelle. Recherche opérationnelle, tome 12, n 3 (1978), p. 325.

<http://www.numdam.org/item?id=RO_1978_12_3_325_2>

(C) AFCET, 1978, tous droits réservés.

L'accès aux archives de la revue « Revue française d'automatique, d'informatique et de recherche opérationnelle. Recherche opérationnelle » implique l'accord avec les conditions générales d'utilisation (http://www.numdam.org/ legal.php). Toute utilisation commerciale ou impression systématique est constitutive d'une infraction pénale. Toute copie ou impression de ce fichier doit contenir la présente mention de copyright.

\section{Numdam}

Article numérisé dans le cadre du programme

Numérisation de documents anciens mathématiques

http://www.numdam.org/ 


\section{LIVRES REÇUS A LA BIBLIOTHĒQUE DE L'A.F.C.E.T.}

J. M. Bouroche, Analyse des données en Marketing, Masson et C $\mathrm{C}^{\mathrm{ie}}$, Paris, 1977.

A. KaUfman et E. Pichat, Méthodes mathématiques non numériques et leurs algorithmes. 1. Algorithmes de recherche des éléments maximaux, Masson et $C^{\mathrm{ie}}$, Paris, 1977.

L. R. SHEnton et K. O. Bowman, Maximum Likelihood Estimation in Small Samples, Griffin's and Co, Londres, 1977.

R. G. Coyle, Management Dynamic Systems, Wiley and Sons, Londres, 1977.

G. E. Whitehouse, B. L. WeChSLER, Applied Operations Research: a Survey, Wiley and Sons, New York, 1976.

M. Roubens, éd., Advances in Operations Research, Proceedings of the EURO II, North Holland, Amsterdam, 1977.

P. W. House, J. MCLeod, Large Scale Models for Policy Evaluation, J. Wiley and Sons, New York, 1977.

E. S. Buffa, J. S. Dyer, Model Formulation and Solution Models, J. Wiley and Sons, New York, 1977.

W. C. Guenther, Sampling Inspection in Statistical Quality Control, Charles Griffin and Cy Ltd, Londres, 1977.

Proceedings of the Annual Reliability and Maintainability Symposium 1977, I.E.E.E. Washington 1977.

W. G. Cochran, Sampling Techniques, John Wiley and Sons, New York, 1977.

R.A.I.R.O. Recherche opérationnelle/Operations Research, vol. 12, n 3, août 1978 